\title{
La dosis de fertilización afecta el rendimiento y calidad en limón Persa (Citrus latifolia Tan.)
}

\author{
Fertilization doses affects yield and quality in Persian lime trees (Citrus latifolia Tan.)
}

\author{
Luis Miguel Conde-Delgado ${ }^{1}$, Iran Alia-Tejacal ${ }^{1 *}$, Luis Alonso Valdez-Aguilar ${ }^{2}$, Rafael Ariza-Flores ${ }^{3}$, \\ Porfirio Juárez-López ${ }^{1}$, Gloria Alicia Pérez-Arias ${ }^{1}$, Clara Pelayo-Zaldivar ${ }^{4}$, \\ Fernando Díaz de León-Sánchez ${ }^{4}$, Arturo Martínez-Morales ${ }^{5}$
}

\section{RESUMEN}

Se evaluó la dosis de restitución 110.6-53.5-28.2 +20, +40, +60 y +100\%, así como árboles de referencia a los que no se fertilizó. El experimento se realizó en árboles de siete años con arreglo de bloques completos al azar. El contenido de clorofilas en las hojas mostró mayor ajuste $\left(\mathrm{r}=0.50-0.81^{*}\right)$ al aumentar las dosis de restitución entre 100 y $160 \%$. El rendimiento fue mayor cuando se aplicó la fórmula de restitución $+40 \%$ con $90.1 \mathrm{~kg}$ árbol-1. La masa del fruto se incrementó significativamente al aplicar los elementos por restitución $+60 \%$ y similar tendencia mostraron el diámetro polar y ecuatorial, y el índice de sabor; no sucedió lo mismo para la acidez titulable, que disminuyó. En conclusión, el limón Persa mostró efecto positivo al aplicar la dosis de restitución y el incremento adicional entre 20 y $60 \%$ de la dosis de restitución.

PALABRAS CLAVE

rendimiento, sólidos soluble totales, color, acidez titulable, fruto

\section{ABSTRACT}

Restitution dose $110.6-53.5-28.2+20,+40,+60 \%$, and $+100 \%$ was evaluated; a group of trees without fertilization completed the experiment. A complete random blocks design in trees of seven years old was evaluated. Chlorophylls lectures in the leaves showed best fit $\left(\mathrm{r}=0.50-0.81^{*}\right)$ when the fertilization doses increased between 100 and 160\%. Yield increase in the restitution doses $+40 \%$ with $90.1 \mathrm{~kg}$ tree $^{-1}$. Fruit mass was increased significantly with the formula of restitution $+60 \%$, similar trends were observed in polar and equatorial diameter and in flavor index. Titratable acidity decreased when Persian lime production dose increased. In conclusion, fertilization with restitution doses and increases from 20, 40 and 60\% are positive in Tahiti lime production.

KEYWORDS

yield, soluble total solids, mass, trititable acidity, fruit

\footnotetext{
${ }^{1}$ Facultad de Ciencias Agropecuarias, Universidad Autónoma del Estado de Morelos. Cuernavaca, Morelos, México.

${ }^{2}$ Departamento de Horticultura, Universidad Autónoma Agraria Antonio Narro. Saltillo, Coahuila, México.

${ }^{3}$ Instituto Nacional de Investigaciones Forestales, Agrícolas y Pecuarias. Iguala de la Independencia, Guerrero México.

${ }^{4}$ Departamento de Ciencias de la Salud, Universidad Autónoma Metropolitana-Iztapalapa. Ciudad de México, México.

${ }^{5}$ División Académica Multidisciplinaria de Jalpa de Méndez, Universidad Juárez Autónoma de Tabasco. Jalpa de Méndez, Tabasco, México.

*Autor para correspondencia. Av. Universidad 1001, col. Chamilpa. 62209 Cuernavaca, Morelos, México.

Correo electrónico: iran.alia@uaem.mx
} 


\section{INTRODUCCIÓN}

La citricultura representa una actividad económica de gran importancia en la fruticultura nacional. Los principales cítricos producidos en México son: naranja, limón Mexicano, limón Persa, toronja y mandarina. En 2016, la superficie establecida con cítricos superó las 556,000 hectáreas (ha), las cuales produjeron cerca de 7.74 millones de toneladas de frutos (SIAP 2017). En el estado de Morelos, los cítricos se introdujeron para su explotación comercial en el año 2000 con 252 ha cultivadas con limón, naranja y mandarina (Alia et al. 2011). En 2016, se registraron 443 ha de limón, 180.8 ha de naranja, 11.2 ha de mandarina y 5 ha de toronja, lo que generó 9,788.3 toneladas de fruta de estos cítricos (SIAP 2017).

El fruto de esta especie se utiliza para extraer jugo fresco, que se emplea como condimento en las comidas y en la preparación de carnes, mariscos, bebidas frías, pasteles, mermeladas, jaleas, nieves y conservas, así como en la preservación de alimentos. De la cáscara o epicarpio, se extrae aceite esencial, que se utiliza en la industria de los cosméticos, mientras que la pulpa sirve para alimentar ganado (Mellado et al. 2015). En México, la mayoría de la producción de limón Persa se exporta a EUA, Japón y a algunos países de Europa (Caamal-Cauich et al. 2014).

La tecnología disponible para cultivar cítricos es muy heterogénea y escasa; además, falta validarla. También hace falta darle una mayor difusión para mejorar la producción y rentabilidad del cultivo de cítricos en el estado de Morelos (Ariza et al. 2009). Curtí-Díaz et al. (1998) mencionan que algunas causas de la baja productividad son: a) desinterés del productor para invertir en su huerta debido a la inestabilidad y predominancia de precios bajos; $b$ ) individualismo para producir y comercializar la fruta; $c$ ) falta de información en la mayoría de los productores acerca de la tecnología existente para elevar la productividad, y d) insuficiente investigación y validación de tecnologías apropiadas a cada región citrícola.

Además del rendimiento, la nutrición mineral puede afectar la calidad del fruto (textura, olor, color, sabor, ausencia de desórdenes en almacenamiento y pudriciones), por lo que se debe relacionar el manejo de ésta con la aceptación por parte del consumidor (Salazar et al. 2006). Se recomienda realizar aplicaciones de nutrimentos en limón Persa considerando los análisis de suelo y dando seguimiento mediante análisis foliar para verificar los avances de la aplicación de los fertilizantes (Mellado et al. 2015). Los estudios que validan la utilización de nutrimentos en el rendimiento y calidad del producto son escasos debido a que los experimentos necesarios se deben realizar a largo plazo. Recientemente, Alia et al. (2012a), realizaron un ensayo de nutrición en limón Persa en Morelos y encontraron escasos efectos de la nutrición en los rendimientos y la calidad del fruto.

La fertilización es la segunda práctica, después del riego en el cultivo de cítricos, para elevar la productividad (Quiñones et al. 2004). El objetivo de la misma en la fase de producción del árbol es obtener la máxima productividad y calidad del fruto, conociendo las necesidades del árbol sin incurrir en excesos o deficiencias. Los análisis de suelo, las hojas y la cantidad de nutrimentos extraídos por el fruto deben considerarse para definir las cantidades de fertilización adicionados al huerto (Salgado-García et al. 2016; Siqueira et al. 2017).

Una nutrición deficiente produce una reducción de la cosecha y en muchos casos del tamaño del fruto. Por su parte, el exceso de fertilizante conduce a consecuencias adversas, entre las que destacan la pérdida de calidad de los frutos, la disminución de la rentabilidad de la plantación, desequilibrios nutricionales por antagonismo con otros elementos, alteraciones difícilmente reversibles de las características físicas o químicas del suelo y contaminación del medio ambiente (Legaz y Primo 1988).

Debido a que los cultivos de cítricos en México se encuentra en expansión, es preciso realizar estudios sobre el manejo adecuado de la nutrición y sobre el manejo poscosecha con el fin de ofrecer a los productores información de utilidad para mejorar el manejo de sus huertas. Por lo anterior, el objetivo de este estudio fue determinar el efecto de la nutrición con nitrógeno, fósforo y potasio en limón Persa cultivado en Morelos, considerando los nutrimentos aportados por el suelo y su efecto en el rendimiento y calidad de los frutos cosechados.

\section{Materiales Y MÉtodos}

El presente trabajo es una continuación de estudios previos sobre nutrición y considera los ensayos y recomendaciones establecidos anteriormente para el desarrollo de fórmulas de fertilización en limón Persa (Alia et al. 2011; Alia et al. 2012b; Maldonado et al. 2001). El experimento se realizó en una huerta comercial de limón Persa localizada en Ticumán,

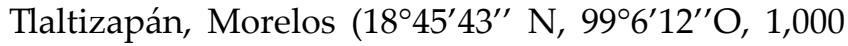
$\mathrm{msnm})$, de clima cálido subhúmedo $\left(\mathrm{Aw}_{\mathrm{o}}\right)$, relieve plano, suelo Feozem calcárico, con profundidad no mayor a $40 \mathrm{~cm}$ y alto contenido de carbonato de 
Cuadro 1. Resultado de análisis de suelo y kilogramos removidos por tonelada de limón Persa cosechada, utilizados en el cálculo de dosis de fertilización en huertas de limón Persa, en Morelos, México.

\begin{tabular}{lllll}
\hline Concepto & $\mathbf{P H}$ & $\mathbf{N}(\%)$ & $\begin{array}{l}\text { K (mg } \\
\left.\mathbf{k g}^{-1}\right)\end{array}$ & $\begin{array}{l}\mathbf{P}(\mathbf{m e q} \\
\mathbf{1 0 0 ~}^{-1} \mathbf{)}\end{array}$ \\
\hline $\begin{array}{l}\text { Análisis de } \\
\text { suelo }\end{array}$ & 7.8 & 0.161 & 3.49 & 0.611 \\
& $\mathrm{~N}(\%)$ & $\mathrm{K}(\mathrm{kg})$ & $\mathrm{P}(\mathrm{kg})$ \\
$\begin{array}{l}\text { Extracción de } \\
\text { elementos en } \\
\text { limón persa }{ }^{2}\end{array}$ & 0.9 & 0.16 & 0.97 \\
\hline $\begin{array}{l}{ }^{1} \text { Resultado de análisis de laboratorio } \\
{ }^{2} \text { kg extraído por tonelada de fruto (Bataglia et al. 1977) }\end{array}$ &
\end{tabular}

Cuadro 2. Tratamientos de fertilización evaluados en limón Persa.

\begin{tabular}{cl}
\hline Tratamiento & \multicolumn{1}{c}{ Dosis De Fertilización (NPK) } \\
\hline T1 & $\begin{array}{l}\text { 00-00-00 (testigo sin fertilización) } \\
\text { 14.4-2.56-15.52 (fertilización por } \\
\text { restitución) } \\
\text { T2 }\end{array}$ \\
T3 & 20.16-3.58-21.72 (restitución $+40 \%)$ \\
T4 & 23.04-4.10-24.83 (restitución $+60 \%)$ \\
T5 &
\end{tabular}

calcio, precipitación y temperatura promedio anuales de $800 \mathrm{~mm}$ y $24{ }^{\circ} \mathrm{C}$, respectivamente (Ambriz et al. 2014). La huerta tenía siete años, con una distancia de plantación de $4 \times 7$ m, y el manejo agronómico se realizó como lo indican Alia et al. (2012b).

Antes de realizar el experimento, se estudió el suelo para conocer la concentración de los elementos minerales y considerarlos en el cálculo de las necesidades nutrimentales (Maldonado et al. 2001). Las muestras de suelo se trasladaron al Laboratorio de Nutrición Vegetal del Colegio de Postgraduados, donde se realizó un análisis del contenido de nitrógeno $(\mathrm{N})$, fósforo $(\mathrm{P})$ y potasio $(\mathrm{K})$. Los resultados fueron utilizados para determinar las fórmulas de fertilización que se habrían de utilizar (cuadro 1), mediante el método de restitución, que consiste en reponer al suelo los nutrientes extraídos por tonelada de fruta tomando en cuenta los nutrientes disponibles en el suelo y el rendimiento esperado de $16 \mathrm{tha}^{-1}$ (Alia et al. 2012a; Maldonado et al. 2001) (cuadro 2). Se utilizó la siguiente fórmula de Salazar (2002):

Requerimientos de elemento $(N, P$ o $K)=$ remoción - aporte del sueloleficiencia del fertilizante
Donde la remoción de los elementos minerales por tonelada de fruta se obtuvo de literatura publicada (Bataglia et al. 1977) y el aporte del suelo se obtuvo a partir de los análisis de suelo realizados (cuadro 1). Se consideró la eficiencia de fertilizante de 65,25 y $80 \%$ para N, P y K (Salazar 2002). Los resultados indicaron que el suelo presentó poco aporte de $\mathrm{N}$ y suficiente de $\mathrm{P}$ y K, por lo que se aplicó lo que extraerá el rendimiento estimado de $16 \mathrm{t} \mathrm{ha}^{-1}$ (Salazar 2002) (cuadro 2).

La aplicación de elementos minerales se realizó en tres fracciones, al inicio se aplicó todo el $\mathrm{P}$ y K y una tercera parte del N (marzo); en dos aplicaciones posteriores se aportó el $\mathrm{N}$ faltante (mayo y julio). Las dosis aplicadas de restitución y de restitución con $20 \%$ a $60 \%$ más de fertilizante, fueron obtenidas para sulfato de amonio (20.5-00-00), fosfato diamónico (18-46-00) y sulfato de potasio (00-00-54); la dosis de fertilización por restitución calculada fue de 110.653.5-28.20 con las fuentes antes indicadas (cuadro 2). Debido a las altas proporciones de carbonatos de calcio en suelo y $\mathrm{pH}$-superiores a 7.1-, se utilizaron las fuentes antes indicadas (cuadro 3). Se utilizó un diseño experimental de bloques completos al azar, con cinco tratamientos. La unidad experimental fue un árbol con seis repeticiones (cuadro 3).

Cuadro 3. Dosis de nutrición (Kg ha $\left.{ }^{-1}\right)$ evaluados en limón Persa.

\begin{tabular}{llll}
\hline Tratamiento & N (SA) & P (DAP) & K (SP) \\
\hline Sin fertilización & 0.0 & 0.0 & 0.0 \\
$\begin{array}{l}\text { Restitución } \\
\begin{array}{l}\text { Restitución } \\
+20 \%\end{array}\end{array}$ & 110.67 & 53.55 & 28.20 \\
$\begin{array}{l}\text { Restitución } \\
+40 \%\end{array}$ & 153.51 & 74.97 & 33.55 \\
$\begin{array}{l}\text { Restitución } \\
+60 \%\end{array}$ & 179.93 & 85.68 & 44.98 \\
\hline
\end{tabular}

$\mathrm{SA}=$ Sulfato de amonio, $\mathrm{DAP}=$ fosfato diamónico y $\mathrm{SP}=$ sulfato de potasio

\section{Variables evaluadas}

Durante el desarrollo del cultivo, se realizaron dos muestreos foliares: uno en enero y otro en mayo. Para esto, se seleccionaron hojas maduras cosechando el quinto foliolo después del que se encuentra en el 
ápice (Curtí-Díaz et al. 1998). Se colectaron 10 hojas por árbol y el follaje se tomó de la parte media del árbol. Las hojas fueron enviadas al Laboratorio de Nutrición Vegetal, del Colegio de Postgraduados, donde se realizó un análisis del contenido de N, P y K.

El contenido de clorofilas se registró en cuatro ocasiones durante el periodo de la evaluación (abril, mayo, julio y agosto). Se utilizó un medidor de clorofila atLEAF (FFGreen LLC, Wilmington, DE) para determinar la actividad máxima de las clorofilas en una longitud de onda de $650 \mathrm{~nm}$. Las lecturas se realizaron en cinco hojas maduras por cada punto cardinal en cada árbol evaluado (Zhu et al. 2002).

Se realizaron seis cosechas entre agosto y octubre; cada una en un intervalo de 15 días. Al final de las cosechas se cuantificó el rendimiento en kilogramos por árbol, para lo cual se utilizó una balanza granataria (Torey ${ }^{\circledR}$, México); los resultados se extrapolaron a una hectárea.

La calidad de los frutos cosechados se determinó tras evaluar la masa del fruto, los componentes del color (luminosidad $\left[\mathrm{L}^{*}\right]$, cromaticidad $\left[\mathrm{C}^{*}\right]$ y ángulo de matiz [h]), los diámetros polar y ecuatorial, el contenido de sólidos solubles totales y acidez titulable, y el índice de sabor. La calidad se evaluó en la cosecha del mes de septiembre.

La masa se cuantificó en 30 frutos seleccionados al azar de los frutos cosechados de cada tratamiento. Para determinar el peso se usó una balanza digital (OHAUS $^{\circledR}$, EUA). En la parte ecuatorial de los mismos frutos y con ayuda de un espectrofotómetro manual (X-rite ${ }^{\circledast}$, Mod. 3960), se realizaron tres lecturas para obtener los datos de $L^{*}\left(0=\right.$ negro y $100=$ blanco); $C^{*}$ ( $0=$ gris y $60=$ puro), y h ( $0=$ rojo púrpura, $90=$ amarillo, $180=$ verde, $270=$ azul, 360=rojo púrpura) (Neguerula 2012). Los diámetros polar y ecuatorial se determinaron en 30 frutos cosechados por tratamiento con ayuda de un vernier digital (Truper ${ }^{\circledR}$, México).

Para determinar el contenido de sólidos solubles totales, cada fruto se seccionó a la mitad y de éste se exprimieron dos gotas sobre un refractómetro PAL-1 (Atago ${ }^{\circledR}$, Japón) a partir de lo cual se registró el contenido de ${ }^{\circ}$ Brix. La acidez titulable (\%) se calculó al extraer $5 \mathrm{~mL}$ de jugo de cada fruto y adicionarle 20 $\mathrm{mL}$ de agua destilada. De esta mezcla se tomaron 5 $\mathrm{mL}$ y se colocaron en un vaso de precipitados donde se adicionaron tres gotas de fenolftaleína $(2 \%)$ y se titularon con $\mathrm{NaOH} 0.1 \mathrm{~N}$ hasta obtener un vire de color rosa (Ladaniya 2008). El índice de sabor se obtuvo al dividir la concentración de sólidos solubles totales y la acidez titulable (Ladaniya 2008).

\section{Análisis de datos}

Los datos fueron sometidos a análisis de varianza y, cuando se encontraron diferencias significativas, se realizó una comparación de medias por el método de Tukey a una probabilidad de 95\%. Los análisis siguieron las indicaciones sugeridas por Castillo (2011) y se llevaron a cabo con ayuda del paquete estadístico $\mathrm{SAS}^{\circledR}$ V. 9.2. Asimismo, se realizaron análisis de regresión polinomial determinando el modelo de mejor ajuste a los tratamientos de nutrición, para lo cual se empleó el programa SigmaPlot (Systat 2010) para la edición de figuras.

\section{RESUltadOS Y DISCUSIÓN}

\section{Dosis de fertilización calculadas}

En un trabajo previo, Alia et al. (2012a) evaluaron una dosis de fertilizante para limón Persa de 159, 32 y 236 $\mathrm{kg}$ de sulfato de amonio, fosfato diamónico y sulfato de potasio, respectivamente, para lo cual consideraron un rendimiento de $17 \mathrm{t} \mathrm{ha}^{-1}$. Entre aquel estudio y el presente trabajo se observó que el $\mathrm{N}$ fue menor en $48.3 \mathrm{~kg}$; el fósforo fue mayor en $20 \mathrm{~kg}$, y el potasio fue 8.36 veces menor. Las diferencias entre las dosis de nutrición determinadas se atribuyen principalmente a que las evaluaciones se realizaron en localidades diferentes.

Se han realizado evaluaciones en limón Persa en Cuba y se reportan dosis de $\mathrm{N}$ entre 106 y $324 \mathrm{~kg}$ de nitrógeno, mientras que para $\mathrm{P}$ y $\mathrm{K}$ se han evaluado dosis de 160 a $250 \mathrm{~kg} \mathrm{ha}^{-1}$ (Hernández 1981). Recientemente, Mellado et al. (2015) determinaron que el limón Persa cultivado en Nayarit, México, tiene extracciones diferenciales de NPK en función del tipo de suelo, época del año y manejo del agua para riego. Así, la remoción de $\mathrm{N}$ y K es mayor en un suelo franco (2.1 y $2.4 \mathrm{~kg} \mathrm{t}^{-1}$ de fruto fresco), en condiciones de riego en primavera; por su parte, la mayor absorción de NPK en invierno fue en suelo migajón arenoso $(2.3,0.38$ y $2.64 \mathrm{~kg} \mathrm{t}^{-1}$ de fruto fresco) y en huertas con riego. Esto sugiere que, para cada explotación comercial de limón Persa, es necesario realizar análisis de suelo con el fin de determinar las fórmulas de fertilización y obtener la máxima producción. Recientemente, Salgado-García et al. (2016) propusieron cuatro dosis de nutrición en la región productora de cítricos en Huimanguillo, Tabasco, entre 184 y $230 \mathrm{~kg}$ de N; 69 a $92 \mathrm{~kg}$ de $\mathrm{P}$, y 240 a $300 \mathrm{~kg}$ de K, en suelos migajón arcilla-arenosa e identificaron 12 subunidades de suelos. 
Cuadro 4. Concentración nutrimental en hoja en función de la dosis de fertilización.

\begin{tabular}{lcccccc}
\hline \multirow{2}{*}{ Tratamiento } & \multicolumn{2}{c}{$\mathbf{N}(\%)$} & \multicolumn{2}{c}{$\mathbf{P}\left(\mathbf{m g ~ L}^{-1}\right)$} & \multicolumn{2}{c}{ K $\left(\mathbf{m g ~ L}^{-1}\right)$} \\
\cline { 2 - 7 } & VI & MM & VI & MM & VI & MM \\
\hline Sin fertilización & 1.83 & $1.64^{\mathrm{az}}$ & 776.2 & $645.54^{\mathrm{a}}$ & 6956 & $5106.7^{\mathrm{a}}$ \\
Restitución & 1.83 & $1.71^{\mathrm{a}}$ & 776.2 & $756.97^{\mathrm{a}}$ & 6956 & $5965.7^{\mathrm{a}}$ \\
Restitución +20\% & 1.83 & $1.81^{\mathrm{a}}$ & 776.2 & $713.10^{\mathrm{a}}$ & 6956 & $5620.6^{\mathrm{a}}$ \\
Restitución +40\% & 1.83 & $1.77^{\mathrm{a}}$ & 776.2 & $689.54^{\mathrm{a}}$ & 6956 & $5300.9^{\mathrm{a}}$ \\
Restitución +60\% & 1.83 & $1.63^{\mathrm{a}}$ & 776.2 & $768.80^{\mathrm{a}}$ & 6956 & $6540.0^{\mathrm{a}}$ \\
DMS & 0.27 & 0.45 & 960.30 & 293.65 & 9824.1 & 2338.1 \\
CV & 5.49 & 15.60 & 46.04 & 24.22 & 52.56 & 24.16 \\
\hline
\end{tabular}

z: letras diferentes en el sentido de las columnas indican diferencias significativas de acuerdo a la prueba de Tukey (0.05)

$\mathrm{VI}=$ valores iniciales antes de aplicar la fertilización; $\mathrm{MM}=$ muestreo en mayo; DMS=diferencia Mínima significativa; CV=coeficiente de variación

\section{Dinámica nutrimental en tejido foliar}

En el muestreo inicial (enero), el contenido de $\mathrm{N}$ en tejido foliar fue de $1.83 \%$, mientras que en el segundo muestreo (mayo), osciló entre 1.63 y $1.81 \%$. Lo anterior indica que la aplicación de la mitad de todo el $\mathrm{N}$ de la dosis calculada no se reflejó en la concentración en las hojas (cuadro 4). Asimismo, no se detectaron diferencias significativas entre las dosis de nutrición en la concentración foliar de $\mathrm{N}$ (cuadro 4).

Los niveles de $\mathrm{P}$ fueron de $776.2 \mathrm{mg} \mathrm{kg}^{-1}$ al inicio del experimento. Posteriormente, en el segundo muestreo (mayo), el contenido del elemento se mantuvo con valores entre 645.54 y $768.80 \mathrm{mg} \mathrm{kg}^{-1}$; no se observaron diferencias significativas entre los tratamientos (cuadro 4). Al igual que en el caso de $\mathrm{N}$ y $\mathrm{P}$, los niveles del elemento al inicio fueron de 6,856 $\mathrm{mg} \mathrm{kg}^{-1} \mathrm{y}$, en el segundo muestreo, los niveles se mantuvieron entre 5,106.7 y 6,540 $\mathrm{mg} \mathrm{kg}^{-1}$ sin mostrar diferencias significativas (cuadro 4).

Gaona (2012) reporta que la concentración de $\mathrm{N}$ en hojas de limón Persa oscila entre 1.3 y $1.4 \%$; la de P entre 1,529 y 1,643 $\mathrm{mg} \mathrm{kg}^{-1}$, y la de potasio entre 2,537 y $2,801 \mathrm{mg} \mathrm{kg}^{-1}$. Además, indica que la aplicación de fertilización incrementó la concentración de $\mathrm{N}$ en la hoja en $1 \%$; el P sólo se incrementó cuando se adicionó la dosis de restitución $+40 \%$, en tanto que el $\mathrm{K}$ no mostró cambios significativos. Hernández (1983) determinó valores de $1.6 \%$ de $\mathrm{N}$ en árboles sin fertilizar durante un periodo de 12 años, lo que indica una productividad baja; sin embargo, al aplicar dosis de 108, 216 y $324 \mathrm{~kg} \mathrm{ha}^{-1}$ de N, la concentración en la hoja se incrementó entre 1.8 y $2.1 \%$ asociado a un incremento en el rendimiento. Se ha reportado que la aplicación de $\mathrm{P}$ en dosis de 180 a $360 \mathrm{~kg} \mathrm{ha}^{-1}$ no favorece el incremento en la concentración de este elemento en tejido foliar.

En el presente experimento no se observó un incremento significativo en los elementos minerales, probablemente porque habían transcurrido dos meses tras haber aplicado la primera fertilización y, posteriormente, ya no se realizaron más análisis de tejido foliar. Sin embargo, se realizaron mediciones de la actividad de clorofilas y se observó que la clorofila en las hojas de limón Persa se incrementó significativamente durante el periodo de evaluación, aunque el ajuste fue mayor conforme se incrementó la dosis de fertilización $\left(\mathrm{r}=0.50^{*}-0.81^{*}\right)$ (figura 1$)$. Jifon et al. (2005) determinaron que el uso de SPAD es buen estimador de la concentración de clorofila $\mathrm{y}$, de forma indirecta, de la concentración de N; es decir: a mayor incremento de los valores, existe mayor concentración de clorofila y $\mathrm{N}$ en las hojas. Así, es necesario realizar curvas de calibración entre las concentraciones de $\mathrm{N}$ y la concentración relativa de clorofila.

\section{Rendimiento}

El rendimiento del fruto fue mayor en la dosis de restitución $+40 \%$, con un promedio por árbol de 90.9 $\mathrm{kg}$; el resto de los tratamientos se mantuvo entre 71.2 y $79.7 \mathrm{~kg}$ (figura 2). El rendimiento aproximado del 


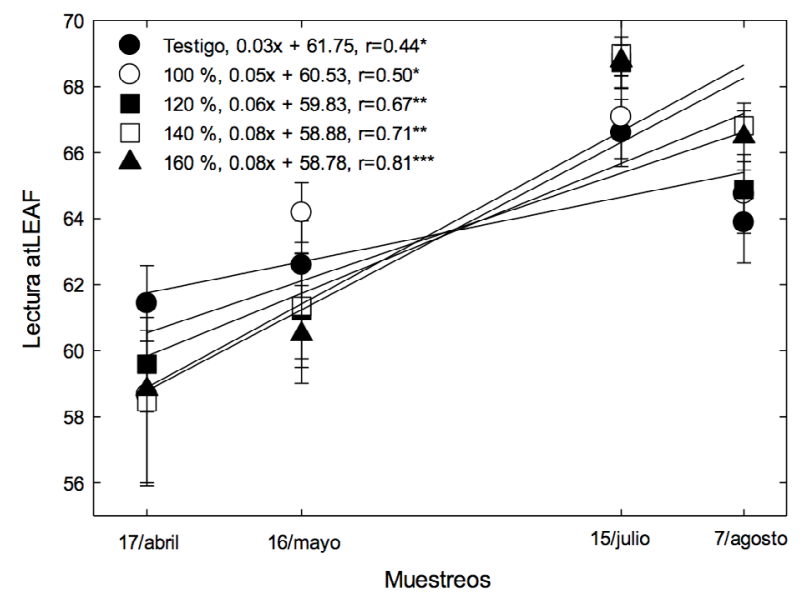

Figura 1. Dinámica de lecturas atLEAF en hojas de limón Persa durante el periodo de evaluación.

tratamiento de restitución $+40 \%$ fue de $32.4 \mathrm{t} \mathrm{ha}^{-1}$ para las cosechas realizadas entre agosto y octubre. Alia et al. (2012a) no encontraron diferencias significativas en el rendimiento en árboles de limón Persa cuando se aplicó la fertilización de restitución mientras que, con incrementos de 20 a 40\%, reportaron un rendimiento promedio entre 20 y $28 \mathrm{~kg}$ por árbol. Lo anterior contrasta con los resultados del presente trabajo, en el cual se obtuvieron rendimientos entre 71.2 y 90.9 $\mathrm{kg}$ por árbol. Esta diferencia probablemente se deba a que el experimento de Alia et al. (2012a) fue realizado en época de menor producción (entre noviembre y junio) en tanto que éste se llevó a cabo entre marzo y octubre, época de mayor producción, con una cosecha en los meses de agosto a octubre.

Machado et al. (2016) reportan que arboles de limón Persa injertados en Volkameriano de siete años produjeron $83.2 \mathrm{~kg}$ árbol ${ }^{-1}$, pero el mejor portainjertos fue la lima Rangpur al producir $90.73 \mathrm{~kg}$ árbol $^{-1}$. Santos et al. (2016) reportan rendimientos entre 28 y $151.9 \mathrm{~kg}$ por árbol en nueve selecciones de limón Persa injertadas sobre Swingle citrumelo de ocho años de edad. Curtí-Díaz et al. (2012) reportan que el limón persa injertado en Volkameriano y de 11 años de edad produjeron en promedio $140.16 \mathrm{~kg}$ árbol ${ }^{-1}$. La diferencia con los rendimientos reportados por otros autores sugiere que, conforme se incrementa la edad de los árboles, el rendimiento también, además de que las condiciones ambientales, el portainjeto y el manejo agronómico afectan la productividad. Los resultados sugieren que en Morelos es necesario incrementar la dosis de nutrición de restitución para mejorar el rendimiento.

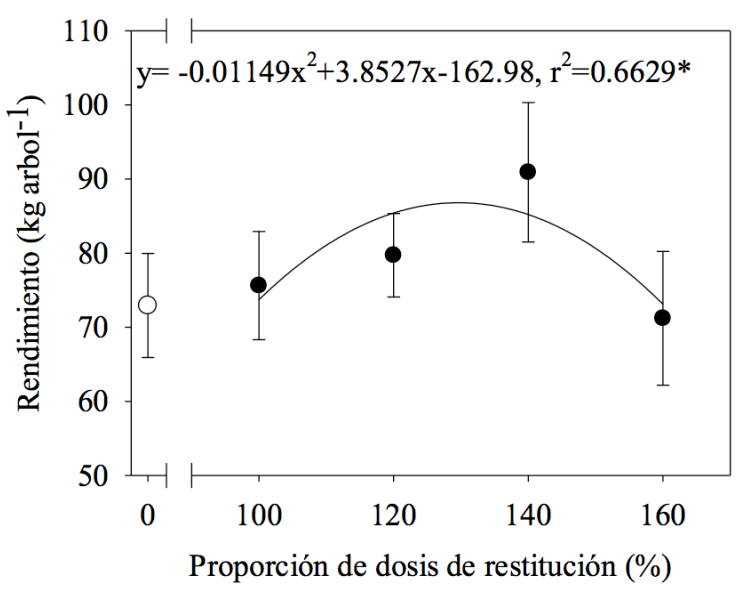

Figura 2. Rendimiento en limón persa fertilizado con diferentes dosis de restitución. Cada punto representa el rendimiento acumulado de seis cortes en seis árboles y su error estándar.

\section{Calidad de frutos}

La masa del fruto se incrementó significativamente conforme la dosis de fertilización. Así, los frutos de los árboles a los que se aplicó la dosis de restitución $+60 \%$ tuvieron entre 7 y 8 g más que aquéllos a los que no se fertilizó o a los que se aplicó sólo la dosis de restitución (figura 3A). Alia et al. (2012a), al evaluar la dosis de restitución 159-32-236 en una huerta de limón Persa, no encontraron efecto en la masa de fruto ya que ésta se mantuvo entre 106.6 y 112.3 g. Por su parte, Castricini et al. (2016) al evaluar dosis de nitrógeno (0 a $\left.100 \mathrm{~kg} \mathrm{ha}^{-1}\right)$ y potasio $\left(0-200 \mathrm{~kg} \mathrm{ha}^{-1}\right)$ no encontraron efecto en la masa del fruto $\mathrm{y}$, en promedio, la masa fue de $86.15 \mathrm{~g}$. Los frutos de limón Persa alcanzaron una masa de $109.2 \mathrm{~g}$ con la mayor proporción de la dosis de restitución (figura $3 \mathrm{~A}$ ).

Los componentes del color mostraron poco cambio en dependencia de la dosis de fertilización que se les aplicó y fueron muy similares a los de los frutos de los árboles que no recibieron fertilización (figura 3B-D). $\mathrm{El}$ color fue verde puro y moderadamente luminoso $\left(L^{*}=56.8\right.$ y $\left.58.8 ; C^{*}=48.7-51.4, h=101.8-102.2\right)$. En este sentido, Alia et al. (2012a) reportan valores muy similares para $\mathrm{L}^{*} \mathrm{y} \mathrm{C}^{*}$, pero cuyo matiz presentó mayor tendencia al verde $(\mathrm{h}=104.5-105.7)$, y no reportan efecto por la aplicación de dosis de restitución +20 o $+40 \%$ de incremento. Castricini et al. (2016), al evaluar diferentes dosis de $\mathrm{N}$ y $\mathrm{K}$, no detectaron efecto en luminosidad $\left(\mathrm{L}^{*}=48.40\right)$ y cromaticidad (39.02); por su parte, encontraron que el matiz disminuyó al incrementar la dosis de $\mathrm{N}$ de $0 \mathrm{~kg}(\mathrm{~h}=120.58)$ a 300 $\mathrm{kg} \mathrm{ha}^{-1}(\mathrm{~h}=120.52)$. En la comercialización del fruto 


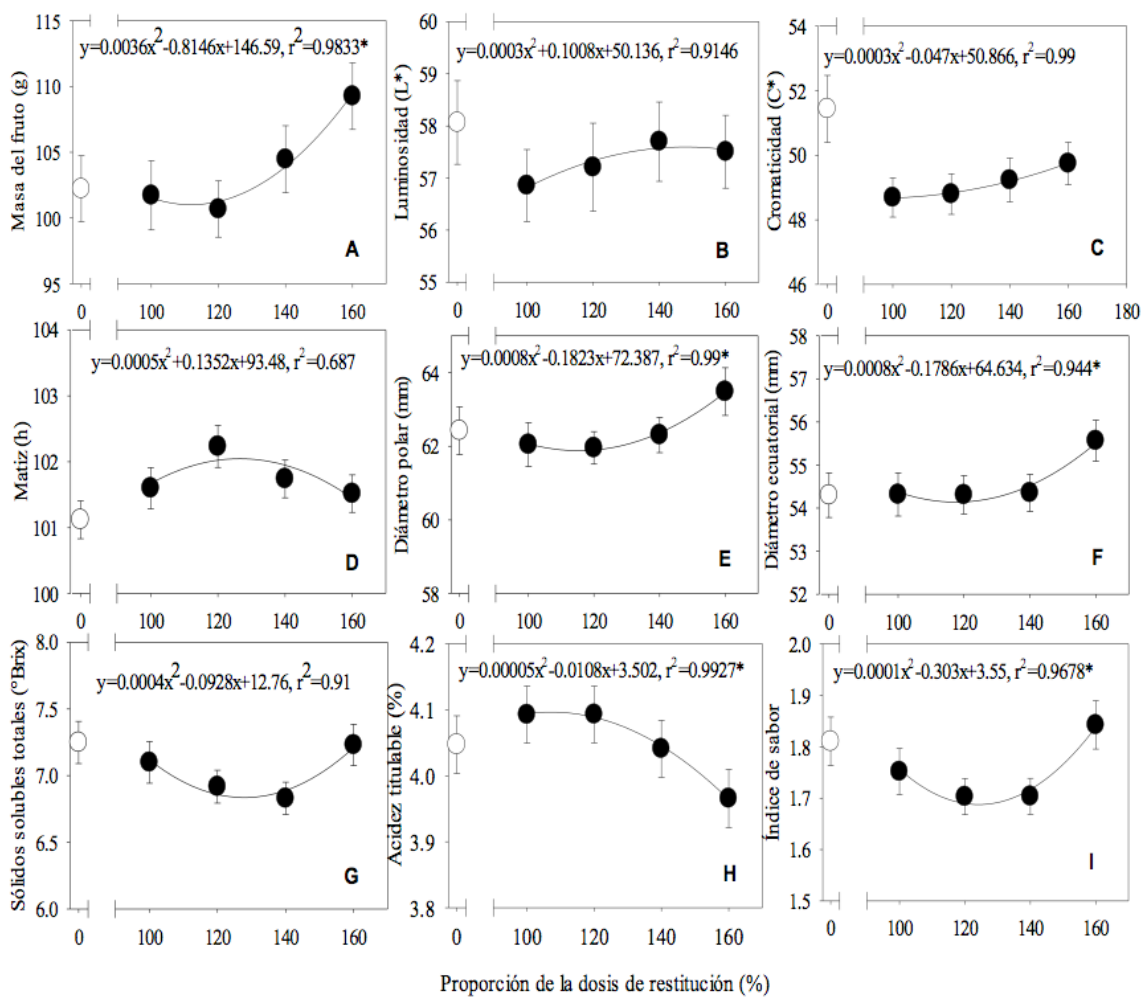

Figura 3. Efecto de la proporción de la dosis de fertilización en calidad de frutos de limón persa. Cada punto representa el promedio de 72 frutos y su error estándar.

de limón persa se prefiere que éste sea de color verde intenso, por lo que las dosis evaluadas no afectaron este parámetro.

El diámetro polar y ecuatorial se incrementaron conforme aumentó la proporción de la dosis de restitución; en general, los frutos tuvieron un diámetro polar entre 62 y $63.5 \mathrm{~mm}$, mientras que el diámetro ecuatorial fue entre 54.3 y $55.5 \mathrm{~mm}$ (figura $3 \mathrm{E}, \mathrm{F})$. Estos valores fueron menores a los reportados por Alia et al. (2012a), quienes encontraron frutos de diámetro polar entre 70.5 y $72.5 \mathrm{~mm}$ y de diámetro ecuatorial entre 70.3 y 74.3; en este último parámetro la dosis de restitución $+20 \%$ mostró los valores mayores. Curtí-Díaz et al. (2012) indican que los calibres comerciales para limón persa son 250 (50-51 $\mathrm{mm}), 230$ (52-53 mm), 200 (54-55 mm), 175 (56-59 mm), $150(59-61 \mathrm{~mm})$ y $110(61-63 \mathrm{~mm})$, donde cada calibre indica la cantidad de frutos con el diámetro indicado que cabe en una caja con capacidad de 40 libras. Así, los frutos evaluados se mantuvieron entre los calibres 200 y 175, lo que significa pueden obtener un mayor precio comparado con los calibres 230 y 250.

No se observó efecto de las diferentes proporciones de la dosis de restitución en el contenido de los sólidos solubles totales, ya que se mantuvieron entre 6.8 y 7.2 ${ }^{\circ}$ Brix (figura 3 G). Alia et al. (2012a) no detectaron efecto de la dosis de restitución +20 o $30 \%$ en el contenido de solidos solubles que se mantuvieron entre 7.2 y 7.9 ${ }^{\circ}$ Brix. Por su parte, Castricini et al. (2016), al evaluar diferentes dosis de $\mathrm{N}$ y K en la calidad de limón Persa, no cuantificaron efecto y reportaron entre 9 y $10{ }^{\circ}$ Brix. La acidez titulable disminuyó significativamente al incrementar la proporción de la dosis de restitución de 100 a $160 \%$ (figura $3 \mathrm{H}$ ). Castricini et al. (2016) indican que, al incrementar la dosis de $\mathrm{K}$ de $0 \mathrm{~kg}$ ha $^{-1}$ año $^{-1}$ (acidez titulable $=5.1 \%$ ) a $200 \mathrm{~kg} \mathrm{ha}^{-1} \mathrm{año}^{-1}$ (acidez titulable $=5.7 \%$ ), ésta se incrementa, lo cual atribuyen a un efecto en el volumen de jugo, donde el incremento en la fertilización de potasio disminuye la concentración de volumen de jugo y esto favorece la concentración de ácidos orgánicos.

El índice de sabor se incrementó con la proporción de restitución de $160 \%$, hasta alcanzar valores de 1.84 (figura 3 I). Almaguer et al. (2017) indican que el limón Persa tiene un índice de sabor promedio de 1.32, muy por debajo del encontrado en el presente trabajo. El índice de sabor indica la dulzura o amargura del fruto, a pesar de que en limas y limones el índice de sabor no es un factor importante como criterio de madurez (Ladaniya 2008). Se observa que la dosis de fertilización ocasionó que el fruto mostrara una tendencia a ser menos amargo. 
Hernández (1983), al evaluar por diez años dosis de fertilización similares en limón Persa cultivado en Cuba, determinó que no hay efecto en las dimensiones del fruto, espesor de cáscara, porcentaje de jugo, contenido de azúcares, acidez titulable y relación entre sólidos solubles y acidez titulable; sólo observó que, al incrementar la dosis del $\mathrm{N}$ y adicionar $\mathrm{P}$ y K, aumentó el contenido de vitamina C. Sin embargo, en la evaluación realizada, la masa del fruto, sus dimensiones, así como la acidez titulable e índice de sabor sí fueron afectadas por las dosis de fertilización evaluadas. Es necesario, sin embargo, realizar estudios que consideren otros factores que podrían interactuar con la nutrición como, por ejemplo, las prácticas agronómicas de poda y riego, entre otros. En este sentido, Fernández et al. (2015), al modelar diferentes escenarios de la producción de limón Persa, determinaron que la nutrición muestra un efecto potencializado cuando se realizan podas previas y existen $50 \mathrm{~mm}$ por mes de lluvia.

\section{LiTERATURA CITADA}

Alia I, Lugo A, Ariza R, Valdez LA, López V, Pacheco P. 2011. Manual de producción en limón Persa y naranja Valencia en el estado de Morelos. Folleto Técnico Núm. 57. Instituto Nacional de Investigaciones Forestales Agrícolas y Pecuarias. Zacatepec, México.

Alia I, Gaona A, Valdez LA, Ariza R, Lugo A, Villegas OG. 2012a. Manejo de nutrición de limón Persa y naranja Valencia en el estado de Morelos. Avances. Folleto Técnico Núm. 57. Instituto Nacional de Investigaciones Forestales Agrícolas y Pecuarias. Zacatepec, México.

Alia I, Goretti M, Ariza R, Lugo A, Andrade M. 2012b. Manejo orgánico de limón Persa y naranja Valencia en el estado de Morelos. Estudio Preliminar. Folleto Técnico Núm. 68. Instituto Nacional de Investigaciones Forestales Agrícolas y Pecuarias. Zacatepec, México.

Almaguer VG, Márquez SR, González V, Cruz N, Ramírez I. 2017. Growth, development and reproductive physiology of the Mexican lime (Citrus aurantifolia Christm (Swingke)). In: Mumtaz MK, Al-Yahyai R, AlSaid F, editors. The lime. Botany, production and uses. Oxfordshire, CABI. p. 54-59.

Ambriz R, Alia I, Ariza R. 2014. Producción de limón persa en época de oferta escasa. Inventio 19: 31-36.

Ariza R, Alia I, Lugo A, Ambriz R, López V. 2009. Manejo agronómico para la producción de naranja 'Valencia' en el estado de Morelos. Folleto para productor 49. Instituto Nacional de Investigaciones Forestales Agrícolas y Pecuarias. Zacatepec, México.

\section{ConCLusiones}

La dosis de fertilización que incluya la restitución $+40 \%$ incrementa el rendimiento en $23 \%$ y mejora la calidad de los frutos de limón Persa, los cuales logran mayor diámetro y peso. Por lo anterior, se estima que la dosis de fertilización óptima para condiciones evaluadas en el estado de Morelos es de 153.5174.97-39.27 de N, P y K.

Bataglia CO, Rodríguez O, Hiroce R, Romano J, Furlani PR, Furlani AMC. 1977. Composição mineral de frutos cítricos na colheita. Bragantia 36: 215-221. https:/doi. org/10.1590/S0006-87051977000100021

Caamal-Cauich I, Jerónimo-Ascencio F, Pat-Fernández VG, Romero-García E, Ramos-García JG. 2014. Análisis de los canales de exportación del limón persa del municipio de Tlapacoyan, Veracruz. Revista Biológico Agropecuaria Tuxpan 2: 183-191.

Castillo LE. 2011. Introducción al SAS® para Windows. Universidad Autónoma Chapingo. Chapingo, México.

Castricini A, Da Silva JTA, Da Silva IP, Rodríguez MGV. 2016. Quality of 'Tahiti' acid lime fertilized with nitrogen and potassium in the semiarid region of Minas Gerais. Revista Brasileira de Fruticultura 39: e-288. https:// doi.org/10.1590/0100-29452017288

Curtí-Díaz SA, Díaz-Zorrilla U, Loredo-Salazar X, Salazar JA, Pastrana L, Rodríguez M. 1998. Manual de producción de naranja para Veracruz y Tabasco. Libro Técnico Núm. 2. Instituto Nacional de Investigaciones Forestales Agrícolas y Pecuarias. San Rafael, México.

Curtí-Díaz SA, Hernández-Guerra C, Loredo-Salazar RX. 2012. Productividad de limón 'persa' injertado en cuatro portainjertos en una huerta comercial de Veracruz, México. Revista Chapingo Serie Horticultura 18: 291305. http://dx.doi.org/10.5154/r.rchsh.2010.11.109. 
Fernández G, Aguilar A, Azzaro-Pantel C, Miranda-Ackerman MA, Purroy R, Pérez MdeR. 2015. Behavior patterns related to the agricultural practices in the production of Persian lime citrus (Citrus latifolia Tanaka) in the seasonal orchard. Computers and Electronics in Agriculture 116: 162-172. https://doi.org/10.1016/j.compag.2015.06.007

Gaona A. 2012. Diagnostico nutrimental y validación de dosis de fertilización en limón persa (Citrus latifolia Tanaka) y naranja 'Valencia' (Citrus sinensis (L.) Osbeck) en el estado de Morelos. Tesis de Maestría en Ciencias. Universidad Autónoma del Estado de Morelos. Cuernavaca, México.

Hernández J. 1981. Efecto del fósforo, potasio y dosis de nitrógeno sobre el rendimiento, calidad del fruto y contenido foliar del naranjo Valencia. Cultivos Tropicales 3: 23-37.

Hernández J. 1983. Influencia de la fertilización con NPK sobre el rendimiento, la calidad del fruto y composición foliar en lima 'Persa'. Cultivos Tropicales 5: 137-147.

Jifon JL, Syvertsen JP, Whaley E. 2005. Growth environment and leaf anatomy affect nondestructive estimates of chlorophyll and nitrogen in Citrus sp. leaves. Journal of the American Society for Horticultural Science 130: 152-158.

Ladaniya SM. 2008. Citrus fruit. Biology, technology and evaluation. Academic Press. San Diego, USA.

Legaz F, Primo ME. 1988. Normas para la fertilización de los agrios. Serie ullets Divulgació Núm. 5-88. Valencia, España. Consellería d'Agricultura i Pesca.

Machado DLM, de Siqueira DL, Salomao LCC, Cecon PR, da Silva DFP. 2016. Evaluation of rootstocks for 'Tahiti' acid lime in northern state of Minas Gerais. Revista Brasileira de Fruticultura 39: e-790. https://doi. org/10.1590/0100-29452017790

Maldonado R, Etchevers JD, Alcántar G, Rodríguez J, Colinas MT. 2001. Estado nutrimental del limón mexicano en suelos calcimorficos. Terra 19: 163-174.

Mellado VA, Salazar S, Álvarez A, Curtí SA. 2015. Remoción de nutrimentos por la cosecha: herramientas para el manejo de la nutrición de limón persa en Nayarit. Folleto Técnico 30. Instituto Nacional de Investigaciones Forestales Agrícolas y Pecuarias. Guadalajara, México.

Neguerula ÁI. 2012. Is the color measured in food the color that we see? In: Caivano JL, Buera MdelP, editors. Color in food. Technological and psychophysical aspect. Buenos Aires, CRC Press. P. 81-91.

Quiñones A, Bañuelos J, Primo M, Legaz F. 2004. Fertilización nítrica en cítricos. III. Dinámica en el sistema planta-suelo del nitrógeno procedente del fertilizante. Levante Agrícola 369: 8-17.

Santos MG, Filho WdSS, Girardi EA, Gesteira AdaS, Passos OS, Ferreira CF. 2016. Initial horticultural performance of nine 'Persian' lime selections grafted onto Swingle citrumelo. Scientia Agricola 73: 109-114. https://doi. org/10.1590/0103-9016-2015-0058
Salazar GS. 2002. Nutrición del aguacate, principios y aplicaciones. Querétaro, México. Instituto Nacional de Investigaciones Forestales Agrícolas y Pecuarias. 165 pp.

Salazar GS., Lazcano I, González JL. 2006. Remoción de nutrimentos por la cosecha de varios cultivares de aguacate en Nayarit. Folleto Técnico Núm. 1. Instituto Nacional de Investigaciones Forestales Agrícolas y Pecuarias. Tepic, México.

Salgado-García S, Palma-López DJ, Zavala-Cruz J, Córdova-Sánchez $S$, Castelán-Estrada $M$, Lagunes-Espinoza LdelC, Ortiz-García CF, Rivera-Cruz MdelC, Ventura-Ulloa F, Marín-Aguilar Á, Moreno-Caliz E, Rincón-Ramírez JA. 2016. Programa de fertilización sustentable para plantaciones de cítricos en Tabasco, México. Ecosistemas y Recursos Agropecuarios 3: 345-356.

[SIAP] Servicio de Información Agroalimentaria y Pesquera. [internet]. 2017. Cierre de la Producción Agrícola 2016. [cited 2017 Sept 23]. Disponible en: https://www. gob.mx/siap/acciones-y-programas/produccion-agricola-33119.

Siqueira DL, Salomao LCC, Aquino CF. 2017. Cultural practices. In: Mumtaz MK, Al-Yahyai R, Al-Said F, editors. The lime. Botany, production and uses. Oxfordshire, CABI. p: 115-134.

Systat. 2010. SigmaPlot®12. User's Guide. San Jose, USA.

Zhu J, Tremblay N, Liang Y. 2002. Comparing SPAD and atLEAF values for chlorophyll assessment in crop species. Canadian Journal of Soil Science 92: 645-648. https://doi.org/10.4141/cjss2011-100 